\title{
A New Method for Image Denoising with Nonlocal Means
}

\section{Xuyao Zhang, Zhiyong Xu}

\begin{abstract}
Denoising is an important problem in image processing, as it can influence the following processing step and decide the final visual effect. To denoise the image and preserve the details, this paper enhances the Nonlocal Means. When estimating the value of a disturbed pixel, we use the information of edges and textures as the weights of pixels involved in calculation, instead of simple Gaussian distance weights. The weights of edge and texture are obtained by direction grads and the coefficients of variation (CV). Finally, based on the results from different images, the enhanced algorithm is shown to have better performance than traditional NLM in SSIM, and has a better visual effect in subjective evaluations.
\end{abstract}

Keywords-denoise; nonlocal means; direction grads; coefficient of variation.

\section{INTRODUCTION}

With the advancement of digital technology, the digital images are replacing conventional images gradually. Unfortunately, digital images are easily to be degraded by different types of noise, while obtained and transmitted. For the reason of noise, the following approaches of image processing are influenced stand a good chance, leading to a bad visual effect. So, it is a hot problem to seek for denoising methods which can be used for image restoration.

One of these conspicuous distortions is additive zeromean Gaussian noise. As for this question, there are many algorithms having some impact. The simple and traditional filter algorithms, such as Gaussian smoothing filter [1] and bilateral filter [2], have been applied widely. This kind of algorithm has a little cost in time and calculation, while the poor performance limits their fields of application. In this situation, more complicated methods come into being. In 1990, the P-M model [3] proposed by Perona and Malik, which was adopted in keeping edge information, was the first one making use of PDE (Partial Differential Equation). Afterward, algorithms based on wavelets [4] became a new trend in image denoising. Owing to the ability of multi-resolution decomposition, wavelets is regarded as a convenient tool, which makes it possible to find an appropriate bourn to remove the noise, as well as keeping more detail information.

In recent years, NLM algorithm [5] comes forth as a new tool of denoising and attracts much attention on account of its superexcellent performance. As a whole, NLM is good enough at processing varied images, but there are still many parts that can be mended. Tasdizen [6] combines NLM with PCA (Principal Component Analysis), putting forward a new algorithm which can improve the ability of resisting distortion by separating

\author{
Institute of Optics and Electronics Chinese Academy of \\ Sciences, Sichuan Province, 610209, China
}

noise and image. Anoosheh [7] changes the weight of similar pixels into a factor containing both ordinary element and edge element. A better melioration is BM3D (Block Matching and 3-D Filtering) [8], which enforce the filtering step by form the image patches into a 3-D matrix, with an outstanding PSNR value.

This paper proposes to enhance the NLM algorithm by adding the account of edge factor, including edge detection and direction grads, and CV factor for texture detection. Different from the traditional NLM and other enhanced algorithm, the method processes an image in 3 kinds of situation. Firstly, in the smooth regions, the weight of filtering factor is similar as original algorithm. Secondly, in the texture regions, the correlative textual pixels will obtain a heavier weight by considering the $\mathrm{CV}$ value. It makes sure that the textual information can be preserved as much as possible. Finally, as for the edge regions, we take the edge detection into account, with the direction grads. If the current pixel and reference one are both in edge regions, which can be judged by an image of edge information, we will calculate the difference of their direction grads in their neighborhoods. It can be used for estimating an exacter weight of edge pixels. Based on the results from different images, our enhanced NLM algorithm is shown to have better performance than traditional NLM in SSIM.

\section{A SIMPLE OVERVIEW OF NLM ALGORITHM}

Denoting the noisy images by $v(i)$, where $i$ is the pixel index. For each pixel, we can estimate the restoration as the following formula:

$$
N L(v)(i)=\sum_{j \in I} w(i, j) v(j)
$$

Where $N L(v)(i)$ is the pixel of the denoised image. $I$ is the searching region of the current pixel $i$, and $j$ is the correlative pixel. $w(i, j)$ denotes the weight of correlative pixel, and it is obtained according to the Gaussian distance of the neighborhoods of pixels:

$$
\begin{aligned}
& w(i, j)=\frac{1}{Z(i)} e^{-\frac{\left\|v\left(N_{i}\right)-v\left(N_{j}\right)\right\|_{2, a}^{2}}{h^{2}}} . \\
& Z(i)=\sum_{j} w(i, j) .
\end{aligned}
$$


Where $v\left(N_{i}\right)$ and $v\left(N_{j}\right)$ are the vectors of the neighborhoods of pixels $i$ and $j$. There will be a heavier weight if the neighborhoods are closer in Euclidian distance. The decay of their Euclidian distance is controlled by the parameter $h \cdot \frac{1}{Z(i)}$ is a parameter of normalization, which is calculated from the sum of all weights of correlative pixels. In a word, a correlative pixel is more similar to the current pixel, and it will get a heavier weight in calculation.

\section{OUR ENHANCED METHOD}

We use the difference of values of pixels instead of Gaussian distance. In an image, the patches with high value of similarity are not only distributing in a small region, as Fig.1. The distribution of pixels is able to show the similarity of two patches better. The difference between the two patches is smaller, and they are more similar visually, no matter how far they are in space.

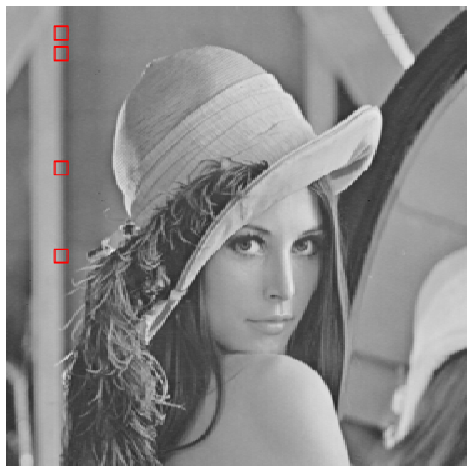

Figure 1. The similar patches

\section{A. The weight of Coefficient of Variation}

Like most of the denoising algorithm, NLM also has the disadvantage of over-smoothing. As it is well known, there is more than one kind of regions in an image. In principle, we can divide an image into 3 parts: smooth region, edge region and textural region. To preserve the information as much as possible, we enhance the NLM basing on finding a factor which can carry out filtering region by region to solve the problem of over-smoothing.

$\mathrm{CV}$ (Coefficient of Variation) is an ingredient distinguishing the smooth and textual region. The method of computing is shown as follows:

$$
C V(i)=\sqrt{\frac{\sigma^{2}\left(N_{i}\right)}{\mu^{2}\left(N_{i}\right)}}=\sqrt{\frac{\frac{1}{\left|N_{i}\right|} \sum_{j \in N_{i}}\left(v(j)-\mu\left(N_{i}\right)\right)^{2}}{\mu^{2}\left(N_{i}\right)}} .
$$

Where $N_{i}$ is the neighborhood of pixel $i$. $\mu$ is the average of pixels in $N_{i}$, and $\sigma^{2}$ is the variance. If one region has more textural information, the value of $\mathrm{CV}$ will become larger. In our denoising algorithm, aiming at making image more flat, we regard $\mathrm{CV}$ as a part of weight, instead of setting a threshold to separate two regions as other algorithm [9].

\section{B. The weight of Edge}

Then we should consider how to keep the edge information, which is the key to ensure a good visual quality. To obtain the information of edge, we build an image in which the values of edge in original image are 1 , the others are 0 . As a standard, this image shows when the weight should be changed by direction grads. Here we adopt the Canny operator in Fig.2.
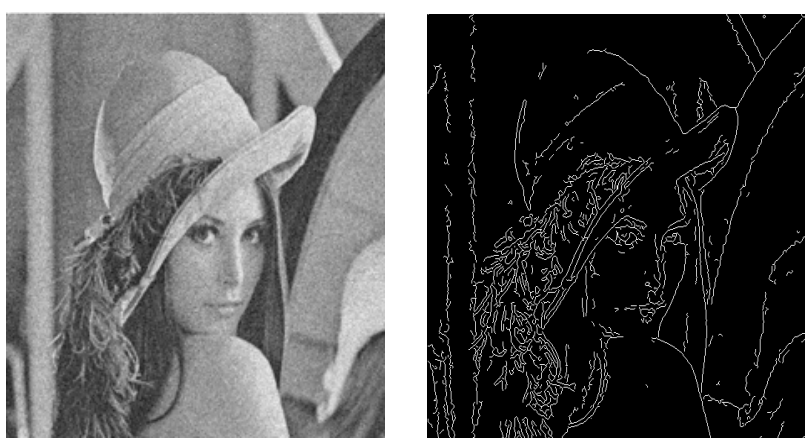

Figure 2. Left: noisy image. Right: image of edge (By choosing suited threshold, we can get the edges with little influence of noise)

After calculating direction grads in an image patches, we can get the transformation of gray in a small region. Usually, it can be expressed in 4 directions. Here we give the horizontal and vertical directions as follows, and the diagonals can be calculated in the same way:

$$
\begin{aligned}
& v\left(N_{v}\right)=|| v(i+1, j)-v(i, j)|+| v(i, j)-v(i-1, j)|-| v(i+1, j)-v(i-1, j) \| . \\
& v\left(N_{h}\right)=\|v(i, j+1)-v(i, j)|+| v(i, j)-v(i, j-1)|-| v(i, j+1)-v(i, j-1)\| .
\end{aligned}
$$

Where $v\left(N_{v}\right), v\left(N_{h}\right)$ and denote the direction grads of vertical and horizontal .For the two diagonals, we use $v\left(N_{45}\right)$ and $v\left(N_{135}\right)$, and $i, j$ are the coordinates of a pixel. In this way, we can descript the information of details in particular.

\section{The weight of direction grads}

Our proposed method aims at the measurement of similarity, considering the grads and configuration instead of calculating Gaussian distance. We change the formula of weight as follows:

$w(i, j)=\frac{1}{Z(i)} e^{-\frac{7 \complement C V\left\|v\left(N_{i}-N_{j}\right)\right\|_{2, a}^{2}+(1-C V)\left\lceil e d g e(i) \llbracket \operatorname{grads}\left(N_{i}, N_{j}\right)\right.}{h^{2}}}$. 
Where $v\left(N_{i}-N_{j}\right)$ is the difference of values of pixels in patches. With the parameters of $7 \sqsubset \mathrm{CV}$ and $(1-C V)$, the smoothing region, where the value of $\mathrm{CV}$ is small, can get a heavier weight, causing a greater filter. On the contrary, in the textual region, the processing of filtering is milder, because of a bigger $\mathrm{CV}$. As for edge region, we have edge( $i$ ) which is a binary image containing pixels of values 1 and 0 . When meeting edges, it is set to 1 , taking the direction grads into account. Otherwise, the value of grads will be ignored, with a 0 value. Besides, $\operatorname{grads}\left(N_{i}, N_{j}\right)$ is an equation:

$$
\begin{array}{r}
\operatorname{grads}(i, j)=\left[\left(v\left(N_{v i}\right)-v\left(N_{v j}\right)\right)^{2}+\left(v\left(N_{h i}\right)-v\left(N_{h j}\right)\right)^{2}\right. \\
\left.+\left(v\left(N_{45 i}\right)-v\left(N_{45 j}\right)\right)^{2}+\left(v\left(N_{135 i}\right)-v\left(N_{135 j}\right)\right)^{2}\right]^{\frac{1}{2}} .
\end{array}
$$

In this equation, we calculate the difference of the direction grads of two patches. It helps us to ensure the similarity of two patches of edge by rule and line.

\section{EXPERIMENTAL RESULT AND ANALYSIS}

In this section, we choose four pictures for the simulation. These pictures are rich in detail information, making the advantage of our algorithm obvious. Here we add Gaussian noise, with 18 and 25 std, to the original images to compare the traditional NLM with our method. Apropos for the quality evaluations, we adopt SSIM. SSIM is acted on the original image and the denoised image. If these images are similar, the value will close to 1 , since its range is from 0 to 1 . In our experiment, we set the parameters $h$ to 6 . The size of similar neighborhood is $3 \times 3$, and the size of search window is $7 \times 7$, through which we get some speedup. The results are shown in Fig.3 and Fig.4.
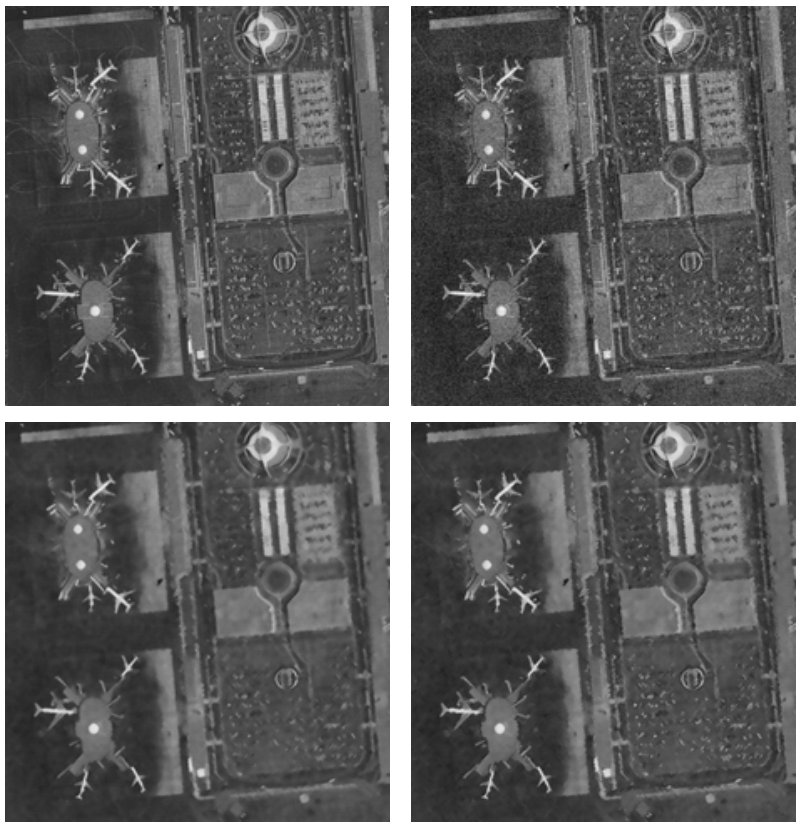

Figure 3. (Left to right, up to bottom) image 'lax' original image, noisy image, denoised by NLM, denoised by our method noise $\mathrm{std}=18$
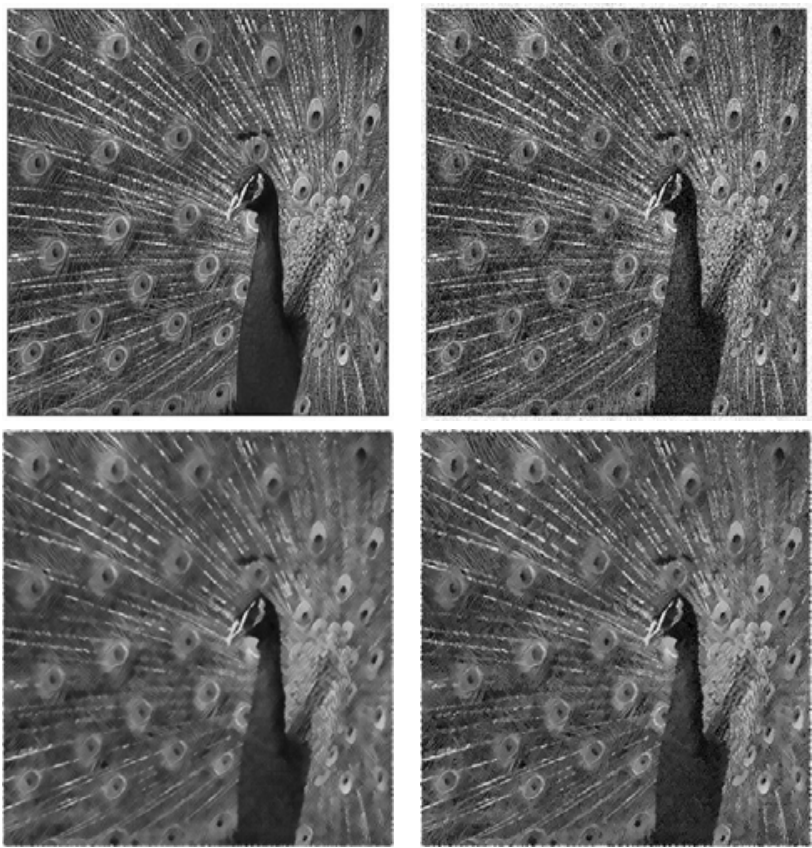

Figure 4. (Left to right, up to bottom) image 'peacock'

original image, noisy image, denoised by NLM, denoised by our method noise $\mathrm{std}=25$

For different intensity of noise, our method shows a preferable result. It can be proved by both of objective evaluations and subjective evaluations. Here we show the objective data of SSIM in Table 1 and Table 2. Besides, we provide the detail edition of images above in Fig. 5 to guarantee the visual effect. 
TABLE 1. THE SSIM OF TESTED IMAGES WITH NOISE STD=18

\begin{tabular}{|c|c|c|c|}
\hline & NLM & $\begin{array}{c}\text { our } \\
\text { method }\end{array}$ & advance \\
\hline baboon & 0.7421 & 0.7869 & $6 \%$ \\
\hline lax & 0.7699 & 0.8134 & $5.7 \%$ \\
\hline peacock & 0.7511 & 0.8318 & $10.7 \%$ \\
\hline cat & 0.5892 & 0.6678 & $13.3 \%$ \\
\hline
\end{tabular}

TABLE 2. THE SSIM OF TESTED IMAGES WITH NOISE STD=25

\begin{tabular}{|c|c|c|c|}
\hline & NLM & $\begin{array}{c}\text { our } \\
\text { method }\end{array}$ & advance \\
\hline baboon & 0.7319 & 0.7731 & $5.6 \%$ \\
\hline lax & 0.7469 & 0.7550 & $1.1 \%$ \\
\hline peacock & 0.7365 & 0.8016 & $8.8 \%$ \\
\hline cat & 0.5738 & 0.6290 & $9.6 \%$ \\
\hline
\end{tabular}
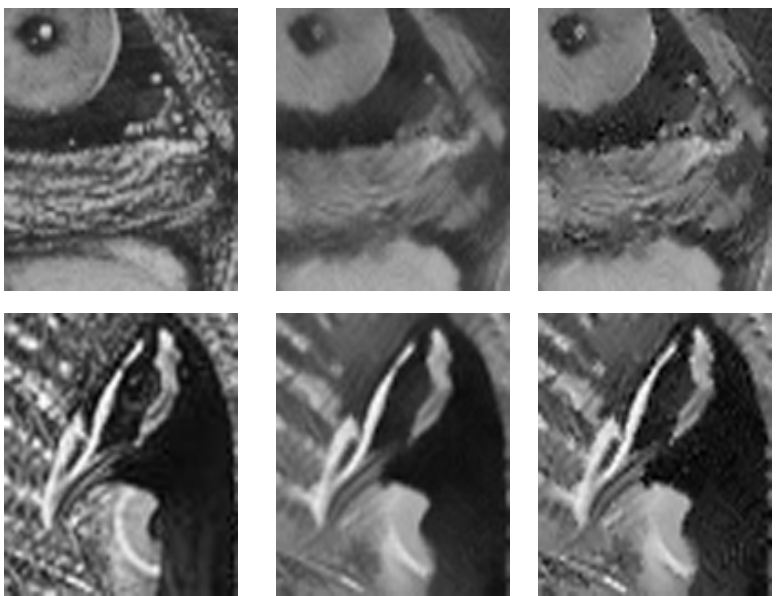

Figure.5 The detail of 'baboon' and 'peacock', noise std=18 (Left to right) original image, denoised by NLM, denoised by our method

In our experiment, the SSIM of our method all have some advances, the value of which can show that our denoised images are more similar to original images than those processed by NLM. From Table 1, we can learn that the advance of our method is decided by the type of image and the intensity of noise. The average of improvement value is $8.9 \%$ when std is equal to 18 , as well as $6.3 \%$, when std is equal to 25 . It's decided by the preservation of texture and edges. As for the whole visual effect, the traditional NLM obtains an over-smoothing outcome, which has lost some detail information. Our method produces an image with abundant edges and texture, resulting in a visual effect with more levels. In Fig.5, we magnify the same patches in the original image and denoised images. In the patches of 'baboon', there are many veins around the eye. According to the edition of NLM, many veins have got lost and the change of gray becomes slight. Comparatively, our method preserves more veins. This situation also exists in image 'peacock'. However, in the smoothing region, the outcome of our method is similar to traditional NLM.

\section{CONCLUSIONS}

This paper puts forward an enhanced image denoising NLM algorithm. It changes the calculation of weight and combines traditional NLM with direction grads and texture detection, possessing a stronger ability of preserving details in an image while denoising. The main idea of our method is to decompose the noisy image and process it depending on the kind of patches. According to objective and subjective result, it shows the improvement in visual effect. However, there are still some questions to be solved. The time cost of NLM is always a big problem in realization. How to speed up our algorithm and how to reduce the pixels used in calculation will be the direction of future study.

\section{REFERENCES}

[1] M. Lindenbaum, M. Fischer, A. M. Bruckstein, On Gabor's contribution to image enhancement, Pattern Recognition, vo1.27, pp.l-8, January 1994.

[2] Tomasi C, Manduchi R. Bilateral filtering for gray and color images[C]. Proceedings of the International Conference on Computer Vision. Bombay, India: IEEE, 1998: 839-846.

[3] Perona P, Malik J. Scale-space and edge detection using an isotropic diffusion [J]. IEEE Transactions on Pattern Analysis and Machines Intelligence, 1990, 12(7):629-639.

[4] L. Sendur and I. W. Selesnick, Bivariate shrinkage functions forwavelet-based denoising exploiting inter scale dependency, IEEE Trans. Signal Process, vol.50, no.11, pp.2744-2756, Nov.2002.

[5] A. Buades, B. Coll, and J. M. Morel, A non-local algorithm for image denoising, in IEEE Computer Society Conference on Computer Vision and Pattern Recognition, 2005, pp. 60-65 vol.2.

[6] Tasdizen T. Principal neighborhood dictionaries for nonlocal means image denoising [J]. IEEE Transactions on Image Processing, 2009, 18(12): 2649-2660.

[7] Anoosheh Heidarzadeh, Alireza Nasiri Avanaki. An Enhanced Nonlocal-Means Algorithm for Image Denoising. IEEE, 2007.

[8] Dabov K, Foi A, Katkovnik V, et al.. Image denoising by sparse 3-D transform-domain collaborative filtering [J]. IEEE Transactions on Image Processing, 2007, 16(8): 2080-2095.

[9] Ying Zhong, Xuezhi Yang, et al.. Non-local Means Denoising Derived from Structure-adapted Block Matching. Journal of Electronics \& Information Technology, vol.35, no.12, pp.29082915 (In Chinese). 